\begin{tabular}{|c|l|}
\hline Title & $\begin{array}{l}\text { Estimation of neutronics parameter sensitivity to nuclear data in random sampling-based uncertainty quantification } \\
\text { calculations }\end{array}$ \\
\hline Author(s) & Chiba, Go; Kawamoto, Y osuke; Tsuji, Masashi; Narabay ashi, Tadashi \\
\hline Citation & $\begin{array}{l}\text { Annals of nuclear energy, 75, 395-403 } \\
\text { https://doi.org/10.1016/.anucene.2014.08.049 }\end{array}$ \\
\hline Issue Date & 2015-01 \\
\hline Doc URL & http://hdl.handle.net/2115/58006 \\
\hline Type & article (author version) \\
\hline File Information & main.r1.pdf \\
\hline
\end{tabular}

Instructions for use 


\title{
Estimation of neutronics parameter sensitivity to nuclear data in random sampling-based uncertainty quantification calculations
}

\author{
Go Chiba*,a $^{*}$, Yosuke Kawamoto ${ }^{\mathrm{a}}$, Masashi Tsuji ${ }^{\mathrm{a}}$, Tadashi Narabayashi ${ }^{\mathrm{a}}$ \\ ${ }^{a}$ Hokkaido University, Kita-ku, Sapporo, Hokkaido 060-8628, Japan
}

\begin{abstract}
We propose a method to estimate sensitivity profiles of neutronics parameters with respect to nuclear data in random sampling-based uncertainty quantification calculations. The proposed method is tested to estimate sensitivity profiles of fast neutron systems criticalities. A high order effect in sensitivity profile estimation is found to be quite important, so a reverse sampling method is developed to mitigate the high order effect. With this reverse sampling method, detail energy group-wise sensitivity profiles can be estimated even though some fluctuations are observed in specific sensitivity profiles. Energy-integrated sensitivity profiles can be accurately calculated with the proposed method.

With the estimated sensitivity profiles, partial uncertainties, that are neutronics parameters uncertainties induced by specific nuclear data uncertainties, are also calculated. Numerical tests reveal that the proposed method reproduces quite well the reference partial uncertainties. A simple and practical partial uncertainty estimation method, which only requires a covariance matrix between neutronics parameter and nuclear data, is also tested and assessed.
\end{abstract}

Key words: sensitivity profile, random sampling, uncertainty quantification

\section{Introduction}

It has been recognized lately that uncertainty quantification for neutronics parameters in nuclear fission reactors are quite important in order to assure their reliability and to improve safety margin (Bratton, 2014). Since one of dominant contributors to neutronics parameters uncertainty is nuclear data, significant efforts have been devoted to evaluations of covariance data for nuclear data and developments of methods for uncertainty propagation calculations from nuclear data to neutronics parameters. The so-called adjointbased uncertainty quantification calculation, in which sensitivity profiles of neutronics parameters with respect to nuclear data are evaluated and neutronics parameters uncertainties are quantified with the simple uncertainty propagation law, has resulted in a great success for static neutronics parameters of fast neutron

\footnotetext{
${ }^{*}$ Corresponding author, Tel: +81-10-706-6683, Fax: +81-10-706-6683

Email address: go_chiba@eng.hokudai.ac.jp (Go Chiba)
} 
reactors. Its application to burnup-dependent parameters and neutronics parameters of thermal neutron reactors, however, is not easy due to complexity of neutronics calculation procedures for those parameters. In such cases the random sampling-based uncertainty quantification calculations are useful, and so many studies have been successfully conducted (Kawano, 2006; Rochman, 2009, 2012; Zwermann, 2012). It is notable that the nuclear data adjustment using integral data has been also realized based on random sampling calculations (Watanabe, 2014).

It has been widely known that sensitivity profiles of neutronics parameters with respect to nuclear data are quite beneficial quantities. In addition to the fact that sensitivity profiles can be used in uncertainty propagation calculations from nuclear data to neutronics parameters as already mentioned, they are quite useful indices for reactor physicists to quantitatively know and specify important nuclear data and their energy range for target neutronics parameters. Furthermore, sensitivity profiles can be utilized for other applications such as representativity factor calculations (Palmiotti, 1984), variance reduction factor calculations (Chiba, 2014), etc.

While sensitivity profiles are essential and calculated in adjoint-based uncertainty quantification calculations, they are not explicitly calculated in random sampling-based uncertainty quantification calculations including the random sampling-based nuclear data adjustment procedure. In the present study, we attempt to estimate detailed sensitivity profiles as by-products in random sampling-based uncertainty quantification calculations.

The present paper is organized as follows; All the theoretical descriptions are provided in Section 2. Section 3 is devoted to describe target neutronics parameters, to which sensitivity profiles are estimated, and employed numerical method/tool in the present study. Numerical results are shown in Section 4 and conclusion of the present study and future perspective are described in Section 5.

\section{Theory}

\subsection{Sensitivity profile estimation}

Let us consider an effective neutron multiplication factor $(k)$ calculation for a nuclear fission reactor using nuclear data $\sigma_{j},(j=1, \ldots, J)$, where $j$ represents a nuclide, a type of reactions and an energy group of the nuclear data. These nuclear data are considered as probability variables in Gaussian distribution, and each of them has its own expectation $\bar{\sigma}_{j}$ and variance $V_{\sigma_{j}}$. Now we produce $I$ sets of nuclear data which are randomly distributed based on their probability distributions, and perform criticality calculations with these nuclear data sets. Then we obtain a statistical distribution for $k$. Its expectation is written as $\bar{k}$ here.

Deviation of $k$ calculated from the $i$ th set of nuclear data, denoted as $k^{i}$, to $\bar{k}$ can be written in the first order approximation as

$$
k^{i}-\bar{k}=\sum_{j}\left(\frac{\partial k}{\partial \sigma_{j}}\right)\left(\sigma_{j}^{i}-\bar{\sigma}_{j}\right) .
$$


Multiplying both sides of this equation by $\sigma_{j^{\prime}}^{i}-\bar{\sigma}_{j^{\prime}}$, summing up for $i$ and dividing them by $I-1$, then we obtain

$$
\begin{aligned}
\frac{1}{I-1} \sum_{i}\left(k^{i}-\bar{k}\right)\left(\sigma_{j^{\prime}}^{i}-\bar{\sigma}_{j^{\prime}}\right) & =\frac{1}{I-1} \sum_{i} \sum_{j}\left(\frac{\partial k}{\partial \sigma_{j}}\right)\left(\sigma_{j}^{i}-\bar{\sigma}_{j}\right)\left(\sigma_{j^{\prime}}^{i}-\bar{\sigma}_{j^{\prime}}\right) \\
& =\sum_{j}\left(\frac{\partial k}{\partial \sigma_{j}}\right) \frac{1}{I-1} \sum_{i}\left(\sigma_{j}^{i}-\bar{\sigma}_{j}\right)\left(\sigma_{j^{\prime}}^{i}-\bar{\sigma}_{j^{\prime}}\right) .
\end{aligned}
$$

As easily understood, this equation can be written with a sample covariance between $k$ and $\sigma_{j^{\prime}}, \operatorname{cov}_{k, \sigma_{j^{\prime}}}$ and that between $\sigma_{j}$ and $\sigma_{j^{\prime}}, \operatorname{cov}_{\sigma_{j}, \sigma_{j^{\prime}}}$ as

$$
\operatorname{cov}_{k, \sigma_{j^{\prime}}}=\sum_{j}\left(\frac{\partial k}{\partial \sigma_{j}}\right) \operatorname{cov}_{\sigma_{j}, \sigma_{j^{\prime}}}
$$

This equation can be written in a simple matrix-vector form as

$$
\mathbf{V}_{k, \sigma}=\mathbf{V}_{\sigma, \sigma} \mathbf{S}_{\sigma}^{k}
$$

where $\mathbf{V}_{k, \sigma}$ is a sample covariance vector between $k$ and $\sigma, \mathbf{V}_{\sigma, \sigma}$ is a sample covariance matrix of nuclear data, and $\mathbf{S}_{\sigma}^{k}$ is a sensitivity vector of $k$ with respect to $\sigma$ and its $j$ th component is defined as $\left(\frac{\partial k}{\partial \sigma_{j}}\right)$. Note that the sample covariance matrix $\mathbf{V}_{\sigma, \sigma}$ is different from a true covariance matrix $\mathbf{V}_{\sigma, \sigma}^{\text {true }}$, which is provided in covariance files in the ENDF evaluation.

If the matrix $\mathbf{V}_{\sigma, \sigma}$ is nonsingular, Eq. (4) can be easily solved and the sensitivity vector is obtained as

$$
\mathbf{S}_{\sigma}^{k}=\mathbf{V}_{\sigma, \sigma}^{-1} \mathbf{V}_{k, \sigma}
$$

Generally the nonsingularity of $\mathbf{V}_{\sigma, \sigma}$ is not assured. In such cases, infinite number of solutions for $\mathbf{S}_{\sigma}^{k}$ are obtained from Eq. (4). In order to obtain a unique solution for $\mathbf{S}_{\sigma}^{k}$ from Eq. (4), we introduce the pseudoinverse of $\mathbf{V}_{\sigma, \sigma}, \mathbf{V}_{\sigma, \sigma}^{\dagger}$, and obtain the following equation:

$$
\mathbf{S}_{\sigma}^{k}=\mathbf{V}_{\sigma, \sigma}^{\dagger} \mathbf{V}_{k, \sigma}
$$

Note that the pseudoinverse matrix is well described in the reference (Mayer, 2000). In this case, the unique sensitivity vector, whose Euclidean norm is minimum among the infinite number of solutions, can be defined.

Equation (6) suggests that sensitivity profiles can be estimated from the sample covariance vector between $k$ and the nuclear data and the sample covariance matrix of the nuclear data. These covariance vector and covariance matrix are obtained in random sampling-based uncertainty propagation calculations.

As already mentioned, the first order approximation is introduced in the derivation of Eq. (6). Thus when a deviation of sampled nuclear data to its expectation, i.e., variance of nuclear data, is large, sensitivity profiles defined in Eq. (6) would be erroneous. If we artificially reduce nuclear data variances, it can be expected that the high order effect in sensitivity profile estimations becomes small. So we prepare 
factorized (reduced) nuclear data covariance data and perform uncertainty quantification calculations for output neutronics parameters $k$. Since the obtained variance for probability distribution of $k$ is also reduced, it is then refactorized and correct variance estimation for $k$ is expected. With this procedure, more accurate sensitivity profiles can be estimated. However, we loose one of merits of the random sampling-based uncertainty quantification calculations: no Gaussian distribution assumption for output parameters.

Here we propose another approach, a reverse sampling method, to diminish the high order effect in sensitivity profile estimations. If we take the second order effect into account, Eq. (1) is modified as

$$
k^{i}-\bar{k}=\sum_{j}\left(\frac{\partial k}{\partial \sigma_{j}}\right)\left(\sigma_{j}^{i}-\bar{\sigma}_{j}\right)+\frac{1}{2} \sum_{j_{1}} \sum_{j_{2}}\left(\frac{\partial k}{\partial \sigma_{j_{1}}}\right)\left(\frac{\partial k}{\partial \sigma_{j_{2}}}\right)\left(\sigma_{j_{1}}^{i}-\bar{\sigma}_{j_{1}}\right)\left(\sigma_{j_{2}}^{i}-\bar{\sigma}_{j_{2}}\right) .
$$

${ }_{34}$ Doing the same procedure as used to obtain Eq. (2), we obtain

$$
\begin{aligned}
& \frac{1}{I-1} \sum_{i}\left(k^{i}-\bar{k}\right)\left(\sigma_{j^{\prime}}^{i}-\bar{\sigma}_{j^{\prime}}\right) \\
= & \sum_{j}\left\{\frac{1}{I-1} \sum_{i}\left(\frac{\partial k}{\partial \sigma_{j}}\right)\left(\sigma_{j}^{i}-\bar{\sigma}_{j}\right)\left(\sigma_{j^{\prime}}^{i}-\bar{\sigma}_{j^{\prime}}\right)\right\} \\
& +\sum_{j}\left\{\frac{1}{I-1} \sum_{i} \frac{1}{2} \sum_{j_{1}} \sum_{j_{2}}\left(\frac{\partial k}{\partial \sigma_{j_{1}}}\right)\left(\frac{\partial k}{\partial \sigma_{j_{2}}}\right)\left(\sigma_{j_{1}}^{i}-\bar{\sigma}_{j_{1}}\right)\left(\sigma_{j_{2}}^{i}-\bar{\sigma}_{j_{2}}\right)\left(\sigma_{j^{\prime}}^{i}-\bar{\sigma}_{j^{\prime}}\right)\right\} .
\end{aligned}
$$

This equation can be written in a matrix-vector form as

$$
\mathbf{V}_{k, \sigma}=\mathbf{V}_{\sigma, \sigma} \mathbf{S}_{\sigma}^{k}+\mathbf{B}
$$

where $\mathbf{B}$ is a vector defined as the second term of the right hand side of Eq.(8).

Now we perform reverse random sampling calculations with $I$ sets of nuclear data in which the $i$ th sample $\hat{\sigma}_{j}^{i}$ is defined as $\hat{\sigma}_{j}^{i}-\bar{\sigma}_{j}=-\left(\sigma_{j}^{i}-\bar{\sigma}_{j}\right)$. As a result of this additional sampling calculations, the following 9 equation can be obtained:

$$
\begin{aligned}
& \frac{1}{I-1} \sum_{i}\left(\hat{k}^{i}-\bar{k}\right)\left(\hat{\sigma}_{j^{\prime}}^{i}-\bar{\sigma}_{j^{\prime}}\right) \\
= & \sum_{j}\left\{\frac{1}{I-1} \sum_{i}\left(\frac{\partial k}{\partial \sigma_{j}}\right)\left(\hat{\sigma}_{j}^{i}-\bar{\sigma}_{j}\right)\left(\hat{\sigma}_{j^{\prime}}^{i}-\bar{\sigma}_{j^{\prime}}\right)\right\} \\
& +\sum_{j}\left\{\frac{1}{I-1} \sum_{i} \frac{1}{2} \sum_{j_{1}} \sum_{j_{2}}\left(\frac{\partial k}{\partial \sigma_{j_{1}}}\right)\left(\frac{\partial k}{\partial \sigma_{j_{2}}}\right)\left(\hat{\sigma}_{j_{1}}^{i}-\bar{\sigma}_{j_{1}}\right)\left(\hat{\sigma}_{j_{2}}^{i}-\bar{\sigma}_{j_{2}}\right)\left(\hat{\sigma}_{j^{\prime}}^{i}-\bar{\sigma}_{j^{\prime}}\right)\right\} \\
= & \sum_{j}\left\{\frac{1}{I-1} \sum_{i}\left(\frac{\partial k}{\partial \sigma_{j}}\right)\left(\sigma_{j}^{i}-\bar{\sigma}_{j}\right)\left(\sigma_{j^{\prime}}^{i}-\bar{\sigma}_{j^{\prime}}\right)\right\} \\
& -\sum_{j}\left\{\frac{1}{I-1} \sum_{i} \frac{1}{2} \sum_{j_{1}} \sum_{j_{2}}\left(\frac{\partial k}{\partial \sigma_{j_{1}}}\right)\left(\frac{\partial k}{\partial \sigma_{j_{2}}}\right)\left(\sigma_{j_{1}}^{i}-\bar{\sigma}_{j_{1}}\right)\left(\sigma_{j_{2}}^{i}-\bar{\sigma}_{j_{2}}\right)\left(\sigma_{j^{\prime}}^{i}-\bar{\sigma}_{j^{\prime}}\right)\right\} .
\end{aligned}
$$

This equation can be written as

$$
\hat{\mathbf{V}}_{k, \sigma}=\mathbf{V}_{\sigma, \sigma} \mathbf{S}_{\sigma}^{k}-\mathbf{B} .
$$


Thus by using Eqs. (9) and (11) we can drop the second order term off and obtain more accurate estimation

for sensitivity vector as

$$
\mathbf{S}_{\sigma}^{k}=\frac{1}{2} \mathbf{V}_{\sigma, \sigma}^{\dagger}\left(\mathbf{V}_{k, \sigma}+\hat{\mathbf{V}}_{k, \sigma}\right)
$$

\subsection{Nuclear data-wise uncertainty estimation}

In the random sampling-based uncertainty quantification, only total uncertainties can be quantified. If sensitivity profiles of output quantities with respect to each nuclear data are available, partial uncertainty induced by uncertainty of specific nuclear data $\tilde{\sigma}$ can be also quantified by using the simple uncertainty propagation law as

$$
V_{\tilde{\sigma}}^{k}=\mathbf{S}_{\tilde{\sigma}}^{k^{T}} \mathbf{V}_{\tilde{\sigma}, \tilde{\sigma}}^{t r u e} \mathbf{S}_{\tilde{\sigma}}^{k},
$$

where $T$ denotes a matrix transpose and $\mathbf{V}_{\tilde{\sigma}, \tilde{\sigma}}^{\text {true }}$ is a true covariance matrix of the specific nuclear data $\tilde{\sigma}$. Furthermore, when the nuclear data $\tilde{\sigma}$ do not have any correlations with other nuclear data, a simple expression can be obtained as follows. If we approximate the sample covariance for $\tilde{\sigma}$ to the true covariance in Eq. (4), Eq. (13) can be transformed as

$$
V_{\tilde{\sigma}}^{k}=\mathbf{S}_{\tilde{\sigma}}^{k^{T}} \mathbf{V}_{k, \tilde{\sigma}}=\left(\mathbf{V}_{\tilde{\sigma}, \tilde{\sigma}}^{\text {true }} \mathbf{V}_{k, \tilde{\sigma}}\right)^{T} \mathbf{V}_{k, \tilde{\sigma}}=\mathbf{V}_{k, \tilde{\sigma}}^{T} \mathbf{V}_{\tilde{\sigma}, \tilde{\sigma}}^{\text {truet}} \mathbf{V}_{k, \tilde{\sigma}}
$$

This expression is quite practical since the partial uncertainty can be quantified only from a sample covariance vector between $k$ and the specific nuclear data. Similar expression for $V_{\tilde{\sigma}}^{k}$ is also available when one applies the reverse sampling method to the sensitivity profile estimations.

\section{Target neutronics parameters and numerical method}

In the present study, we attempt to estimate sensitivity profiles of criticality (the effective neutron multiplication factor) with respect to nuclear data for the following three fast neutron systems. The geometrical specification and nuclide number densities of the compositions are taken from the ICSBEP handbook (Briggs, 2006). The notations adopted in the ICSBEP handbook are also shown in the following list.

- Jezebel (PU-MET-FAST-001): A bare sphere of plutonium (95at\% Pu-239).

- Godiva (HEU-MET-FAST-001) : A bare sphere of high enriched (94wt\%) uranium.

- Flattop-25 (HEU-MET-FAST-028): A high enriched uranium sphere surrounded by a normal uranium reflector.

All the numerical calculations are performed with a multi-purpose reactor physics calculation code system $\mathrm{CBZ}$, which is being developed at Hokkaido university. The CBZ code system has been well validated through a post-irradiation examination analysis (Kawamoto, 2012). 
A 70-group neutron cross section library is generated from JENDL-4.0 (Shibata, 2011) by the NJOY99 code (MacFarlane, 2010). A lethargy width of all the energy groups except for the final one (from $10^{-5} \mathrm{eV}$ to $0.322 \mathrm{eV}$ ) is 0.25 . Covariance data of uranium-235, -238 and plutonium-239 nuclear data given in JENDL-4.0 are also processed by NJOY-99 into the 70-group structure. Note that correlations between different isotopes and different reactions are neglected for simplifications. Criticality calculations (wholecore neutron transport calculations) are performed by a discrete ordinates neutron transport solver of CBZ. Scattering anisotropy is considered up to the first Legendre moment and the 16-point double Gaussian angular quadrature set is used.

Reference sensitivity profiles are calculated by the perturbation theory using forward and adjoint neutron fluxes in the same 70-group structure.

\section{Numerical results}

In the subsection 2.1, covariance data and sensitivity profiles are defined as absolute values for simplicity. On the other hand, in this section, all these quantities are defined as relative values. A sensitivity of $k$ with respect to $\sigma$ is defined as $\frac{\partial k}{\partial \sigma} \cdot \frac{\sigma}{k}$.

\subsection{Sample covariance vs. true covariance}

In this subsection and the following subsections 4.2 and 4.3 , we estimate sensitivity profiles of the Godiva criticality to uranium-235 cross sections. For simplification, we only consider covariance data for only the following three nuclear data: fission cross section $\sigma_{f}$, capture cross section $\sigma_{c}$ and an averaged number of emitted neutrons per a fission reaction $\bar{\nu}$.

As shown in Eq. (6), sensitivity profiles can be estimated from the sample covariance of nuclear data. First we observe dependence of accuracy of estimated sensitivity profiles on the number of samples. Figure 1 shows sensitivity profiles of the Godiva criticality to uranium-235 fission cross section calculated with Eq. (6). The y-scale is multiplied by 0.25 since the lethargy width of all the energy groups shown in this figure is 0.25 . While sensitivity profile estimations with 100 samples result in some fluctuations in whole energy range, sensitivity profiles estimated from 400 samples agree quite well with the references and no fluctuations are observed.

If the true covariance matrix of nuclear data can be used instead of the sample covariance, it would be better from a view point of computational burden since we do not have to calculate and store the sample covariance matrix for nuclear data when performing random sampling calculations. Figure 2 shows sensitivity profiles estimated from the true covariance matrix of nuclear data. Even if 6,400 samples are considered, it is difficult to reproduce the reference sensitivity profiles by the random sampling-based method. It is due to a large difference between a sampled covariance matrix and a true one. 


\subsection{Covariance factorization to mitigate high order effect}

As already described, the first order approximation is introduced to derive Eq. (6), so this approximation sometimes deteriorates sensitivity profiles estimation. While it has been shown in the preceding subsection that the Godiva criticality sensitivity to uranium-235 fission cross section is well estimated, sensitivity to uranium-235 capture cross section is not well reproduced as fission cross section. Since this poor accuracy would be caused by the high order effect, all the elements of the covariance matrices are multiplied by $f^{2}$, which is less than unity, and the sensitivity profile estimations are carried out with the proposed technique with 400 samples. Figure 3 shows sensitivity profiles of the Godiva criticality to uranium-235 capture cross section. Fluctuations are reduced when small values of $f$ are used because the high order effect is mitigated.

\subsection{Reverse sampling method to mitigate high order effect}

The covariance factorization tested in the preceding subsection has a demerit and the reverse sampling method is more preferable as described in subsection 2.1 because the reverse sampling method does not change the nuclear data covariance matrices. Figure 4 shows sensitivity profiles of the Godiva criticality to uranium-235 capture cross section obtained with or without the reverse sampling method with 400 samples. Note that when the reverse sampling method is employed, 200 samples and their reverse 200 samples are used and the total number of samples is 400 . As shown in this figure, the employment of the reverse sampling method provides accurate sensitivity profiles without any fluctuations. This result shows that the reverse sampling method works quite well to mitigate the high order effect and is promising for sensitivity profile estimations.

\subsection{Sensitivity profile estimations in random sampling calculations with reverse sampling method}

In this subsection, sensitivity profiles of criticalities of Godiva, Jezebel and Flattop-25 with respect to six types of nuclear data are estimated. In addition to $\sigma_{f}, \sigma_{c}$ and $\bar{\nu}$, we consider the following three types of nuclear data: elastic scattering cross section $\sigma_{e}$, inelastic scattering cross section $\sigma_{i e}$ and averaged cosine of scattering angle of elastic scattering $\bar{\mu}$. Two bare fast systems, Godiva and Jezebel, consist of unique fissile material, so covariance matrices for six nuclear data are considered in these calculations. On the other hand, since Flattop-25 is a highly-enriched uranium core surrounded by depleted uranium, twelve nuclear data, six for uranium-235 and six for uranium-238, are considered. In other words, we prepare sets of cross section data in which twelve nuclear data are randomly determined. We use the 70-group structure for nuclear data representation, so the sizes of the whole covariance matrices for nuclear data are 420 for Godiva and Jezebel and 840 for Flattop-25. For consistent comparisons in sensitivity profiles, we use 400 samples for Godiva and Jezebel calculations and 800 samples for Flattop-25 calculations. The reverse sampling method is employed to all the calculations in this subsection. 
Figure 5 shows sensitivity profiles of Godiva criticality to uranium-235 nuclear data. For all the six nuclear data, the proposed method reproduces well the reference sensitivity profiles with some fluctuations. In comparison with the sensitivity profile shown as 'w reverse sampling' in Fig. 4, the sensitivity profile to capture cross section shown in Fig. 5 has more and larger fluctuations. This difference comes from a fact that the sensitivity profile shown in Fig. 5 is obtained from random sampling calculations considering uncertainties for six nuclear data while that in Fig. 4 is obtained considering uncertainties only for three nuclear data. This result suggests that when one considers uncertainties of a larger number of nuclear data, a larger number of samples are required to obtain accurate sensitivity profiles.

Figure 6 shows sensitivity profiles of Jezebel criticality to plutonium-239 nuclear data. Whereas sensitivity profiles to $\sigma_{f}, \bar{\nu}, \sigma_{i e}$ and $\bar{\mu}$ are successfully calculated by the proposed method as the Jezebel case, sensitivity profiles to the other two nuclear data, $\sigma_{c}$ and $\sigma_{e}$, show larger fluctuation than the Jezebel case. At present this difference has not yet been well explained, but it would come from the structure of the covariance matrices of nuclear data. Further investigations with much more calculation cases are required in future.

Figures 7 and 8 show sensitivity profiles of Flattop-25 criticality to uranium-235 and uranium-238 nuclear data respectively. On some nuclear data such as elastic cross section and $\bar{\mu}$ of uranium-235 and $\bar{\nu}$ of uranium-238, the proposed method provides sensitivity profiles with poor accuracy. Generally it gives reasonable results.

Table 1 summarizes energy-integrated sensitivity profiles calculated by the proposed method and their differences to the reference. Whereas the energy group-wise sensitivity profiles show some fluctuations, energy-integrated sensitivities are well calculated by the proposed method.

\subsection{Estimation of partial uncertainty}

As described in subsection 2.2, partial uncertainty, that is induced by specific nuclear data uncertainty, can be evaluated if sensitivity profiles are available. Using sensitivity profiles obtained in the preceding section with 400 samples and the reverse sampling method, the partial uncertainties are calculated based on Eq. (13). Reference partial uncertainties are obtained from the sandwich rule using the sensitivity profiles obtained with the perturbation theory. Numerical results are shown in Table 2. On all the partial uncertainties, the present method based on Eq. (13) with 400 samples reproduces quite well the reference uncertainties.

In addition to the method based on Eq. (13), another method based on Eq. (14), which requires only sample covariance matrix between output quantity and nuclear data, is also attempted to calculate partial uncertainties. Two calculations, one with 400 samples and the other with 1,600 samples, are carried out. The results are shown also in Table 2. When the number of samples is small, the method based on Eq. (14) does not reproduce well the reference partial uncertainties. If the number of samples is increased, the accuracy 
of the method based on Eq. (14) is improved. However, even if 1,600 samples are used, better consistency in the partial uncertainties cannot be obtained by the method based on Eq. (14) than the method based on Eq. (13). As shown in the previous section, this is due to a difference between the true covariance matrix and the sample covariance matrix of nuclear data. Since this method is quite practical, it would be beneficial to grasp a degree of partial uncertainties.

\section{Concluding Remarks}

Random sampling-based uncertainty quantification for neutronics parameters of nuclear fission reactors has been widely and successfully applied in reactor physics field. In the present paper, we have newly proposed a method to estimate sensitivity profiles of neutronics parameters with respect to nuclear data in random sampling-based uncertainty quantification calculations. The proposed method has been tested to estimate sensitivity profiles of fast neutron systems criticalities. It has been found that a high order effect in sensitivity profiles estimations is quite important, so we have developed a reverse sampling method to mitigate the high order effect. With this reverse sampling method, detailed energy group-wise sensitivity profiles can be estimated in random sampling calculations even though some fluctuations have been observed in specific sensitivity profiles. Energy-integrated sensitivity profiles can be accurately calculated with the proposed method.

With the estimated sensitivity profiles, partial uncertainties, that are neutronics parameters uncertainties induced by specific nuclear data uncertainties, have been also calculated. Numerical tests have revealed that the proposed method has reproduced quite well the reference partial uncertainties calculated with the large number of samples. A simple and practical partial uncertainty estimation method, which only requires a covariance matrix between neutronics parameter and nuclear data, has been also tested and assessed.

The following subjects still remain for future studies.

- When one considers various kinds of nuclear data, the size of the nuclear data covariance matrix becomes huge and a large computer memory to store the sample covariance matrix is required. One should remind that correlations exist among different nuclear data even if such correlations are not given in the original nuclear data.

- It is required to quantitatively estimate how reliable estimated sensitivity profiles are.

- The efficiency of the reverse sampling method should be further discussed from a view point of estimation of statistical information through random sampling. 


\section{Acknowledgements}

The authors are grateful to Profs. Akio Yamamoto and Tomohiro Endo of Nagoya University for their advices on this study. This work was supported by JSPS KAKENHI Grant Number 24561040.

\section{References}

Bratton, R.N., Avramova, M., Ivanov, K., 2014. OECD/NEA benchmark for uncertainty analysis in modeling (UAM) for LWRs - summary and discussion of neutronics cases (phase I), Nucl. Eng. Technol., 46, 313-342.

Briggs, J.B. (Ed.), 2006. International handbook of evaluated criticality safety benchmark experiments, NEA/NSC/DOC (95)03/I, Nuclear Energy Agency, Paris (2006 Edition).

Chiba, G., Tsuji, M., Narabayashi, T., 2014. Variance reduction factor of nuclear data for integral neutronics parameters, Nucl. Data Sheets, (submitted).

Kawamoto, Y., Chiba, G., Tsuji, M., Narabayashi, T., 2012. Validation of CBZ code system for post irradiation examination analysis and sensitivity analysis of (n, $\gamma$ ) branching ratio, Proc. of the 2012 Annual Symposium on Nuclear Data (NDS 2012), Kyoto, Japan, Nov. 15-16, 2012, [to be published].

Kawano, T., Hanson, K.M., Frankle, S., Talou, P., Chadwick, M.B., Little, R.C., 2006. Evaluation and propagation of the ${ }^{239} \mathrm{Pu}$ fission cross section uncertainties using a Monte Carlo technique, Nucl. Sci. Eng., 153, 1-7.

MacFarlane, R. E., Kahler, A. C., 2010. Methods for processing ENDF/B-VII with NJOY, Nucl. Data Sheets, 111, 2739-2890.

Mayer, C., 2000. Matrix analysis and applied linear algebra, Society for industrial and Applied Mathematics, Philadelphia.

Palmiotti, G., Salvatores, M., 1984. Use of integral experiments in the assessment of large liquid-metal fast breeder reactor basic design parameters, Nucl. Sci. Eng., 87, 333-348.

Rochman, D., Koning, A.J., Van Der Marck, S.C., 2009. Uncertainties for criticality-safety benchmarks and $k_{e f f}$ distributions, Ann. Nucl. Energy, 38, 942-952.

Rochman, D., Koning, A.J., Da Cruz, D.F., 2012. Propagation of $235,236,238 \mathrm{U}$ and ${ }^{239} \mathrm{Pu}$ nuclear data uncertainties for a typical PWR fuel element, Nucl. Technol., 179, 323-338.

Shibata, K., Iwamoto, O., Nakagawa, T., Iwamoto, N., Ichihara, A., Kunieda, S., Chiba, S., Furutaka, K., Otuka, N., Ohsawa, T., Murata, T., Matsunobu, H., Zukeran, A., Kamada, S., Katakura, K., 2011. JENDL-4.0: a new library for nuclear science and engineering, J. Nucl. Sci. Technol., 48, 1-30.

Watanabe, T., Endo, T., Yamamoto, A., Kodama, Y., Ohoka, T., Ushio, T., 2014. Cross section adjustment method based on random sampling technique, J. Nucl. Sci. Technol., 51, 590-599.

Zwermann, W., Krzykacz-Hausmann, B., Gallner, L., Klein, M., Pautz, A., Velkov, K., 2012. Aleatoric and epistemic uncertainties in sampling based nuclear data uncertainty and sensitivity analyses, Proc. of Int. Conf. of Physic of Reactor, Physor 2012, Knoxville, TN, April 15-20, 2012. 
(a) Godiva criticality to uranium-235 nuclear data

\begin{tabular}{ccccc}
\hline & $>1.05 \mathrm{MeV}$ & $0.111-1.05 \mathrm{MeV}$ & $0.012-0.111 \mathrm{MeV}$ & $<0.012 \mathrm{MeV}$ \\
\hline$\sigma_{f}$ & $+0.323\left(-0.012^{*}\right)$ & $+0.304(+0.006)$ & $+0.034(+0.005)$ & $+0.001(+0.000)$ \\
$\sigma_{c}$ & $-0.005(+0.000)$ & $-0.026(+0.000)$ & $-0.008(-0.001)$ & $+0.000(+0.001)$ \\
$\bar{\nu}$ & $+0.477(-0.003)$ & $+0.438(-0.015)$ & $+0.053(+0.000)$ & $+0.003(+0.001)$ \\
$\sigma_{e}$ & $+0.027(-0.001)$ & $+0.063(-0.006)$ & $+0.017(+0.008)$ & $-0.004(-0.004)$ \\
$\sigma_{i e}$ & $+0.044(-0.001)$ & $+0.039(+0.001)$ & $-0.002(-0.002)$ & $+0.000(+0.000)$ \\
$\bar{\mu}$ & $-0.074(+0.000)$ & $-0.038(+0.000)$ & $+0.000(+0.001)$ & $+0.001(+0.001)$ \\
\hline
\end{tabular}

${ }^{*}$ Difference to reference.

(b) Jezebel criticality to plutonium-239 nuclear data

\begin{tabular}{ccccc}
\hline & $>1.05 \mathrm{MeV}$ & $0.111-1.05 \mathrm{MeV}$ & $0.012-0.111 \mathrm{MeV}$ & $<0.012 \mathrm{MeV}$ \\
\hline$\sigma_{f}$ & $+0.461(-0.007)$ & $+0.252(-0.001)$ & $+0.018(+0.000)$ & $-0.002(-0.003)$ \\
$\sigma_{c}$ & $-0.001(+0.000)$ & $-0.009(-0.003)$ & $+0.003(+0.005)$ & $-0.003(-0.003)$ \\
$\bar{\nu}$ & $+0.590(-0.027)$ & $+0.339(+0.005)$ & $+0.022(+0.001)$ & $-0.005(-0.005)$ \\
$\sigma_{e}$ & $+0.020(+0.002)$ & $+0.035(-0.005)$ & $+0.007(+0.003)$ & $-0.012(-0.012)$ \\
$\sigma_{i e}$ & $+0.026(-0.001)$ & $+0.012(-0.001)$ & $+0.005(+0.005)$ & $-0.002(-0.002)$ \\
$\bar{\mu}$ & $-0.074(-0.001)$ & $-0.030(-0.001)$ & $+0.000(+0.000)$ & $-0.001(-0.001)$ \\
\hline
\end{tabular}

(c) Flattop-25 criticality to uranium-235 nuclear data

\begin{tabular}{ccccc}
\hline & $>1.05 \mathrm{MeV}$ & $0.111-1.05 \mathrm{MeV}$ & $0.012-0.111 \mathrm{MeV}$ & $<0.012 \mathrm{MeV}$ \\
\hline$\sigma_{f}$ & $+0.236(-0.012)$ & $+0.293(-0.002)$ & $+0.046(-0.001)$ & $-0.002(-0.003)$ \\
$\sigma_{c}$ & $-0.004(+0.000)$ & $-0.031(+0.000)$ & $-0.012(+0.001)$ & $+0.000(+0.001)$ \\
$\bar{\nu}$ & $+0.358(-0.010)$ & $+0.453(-0.015)$ & $+0.087(-0.001)$ & $+0.003(+0.001)$ \\
$\sigma_{e}$ & $+0.015(+0.001)$ & $+0.018(-0.003)$ & $-0.002(-0.002)$ & $+0.002(+0.002)$ \\
$\sigma_{i e}$ & $+0.014(+0.000)$ & $+0.020(+0.000)$ & $+0.000(+0.000)$ & $+0.000(+0.000)$ \\
$\bar{\mu}$ & $-0.030(+0.004)$ & $-0.006(+0.006)$ & $-0.005(-0.005)$ & $+0.004(+0.004)$ \\
\hline
\end{tabular}

(d) Flattop-25 criticality to uranium-238 nuclear data

\begin{tabular}{ccccc}
\hline & $>1.05 \mathrm{MeV}$ & $0.111-1.05 \mathrm{MeV}$ & $0.012-0.111 \mathrm{MeV}$ & $<0.012 \mathrm{MeV}$ \\
\hline$\sigma_{f}$ & $+0.057(-0.004)$ & $+0.000(+0.000)$ & $+0.000(+0.000)$ & $-0.001(-0.001)$ \\
$\sigma_{c}$ & $-0.002(+0.000)$ & $-0.030(-0.001)$ & $-0.014(+0.001)$ & $+0.000(+0.001)$ \\
$\bar{\nu}$ & $+0.052(-0.030)$ & $+0.009(+0.008)$ & $+0.006(+0.006)$ & $+0.013(+0.013)$ \\
$\sigma_{e}$ & $+0.017(+0.000)$ & $+0.104(-0.009)$ & $+0.021(+0.005)$ & $+0.000(+0.000)$ \\
$\sigma_{i e}$ & $+0.036(-0.003)$ & $+0.036(+0.000)$ & $-0.002(-0.001)$ & $+0.000(+0.000)$ \\
$\bar{\mu}$ & $-0.065(+0.002)$ & $-0.049(+0.002)$ & $-0.002(-0.001)$ & $+0.001(+0.001)$ \\
\hline
\end{tabular}


Table 2: Nuclear data-wise uncertainty in criticalities in unit of $\% \Delta k / k k^{\prime} . I$ denotes the number of samples.

(a) Godiva criticality to uranium-235 nuclear data

\begin{tabular}{ccccc}
\hline & Reference & Eq. (13) & \multicolumn{2}{c}{ Eq. (14) } \\
& & $(I=400)$ & $(I=400)$ & $(I=1,600)$ \\
\hline$\sigma_{f}$ & 0.32 & 0.32 & 0.54 & 0.38 \\
$\sigma_{c}$ & 0.21 & 0.21 & 0.50 & 0.28 \\
$\bar{\nu}$ & 0.28 & 0.27 & 0.40 & 0.37 \\
$\sigma_{e}$ & 0.43 & 0.43 & 0.58 & 0.51 \\
$\sigma_{i e}$ & 0.66 & 0.66 & 0.77 & 0.68 \\
$\bar{\mu}$ & 0.41 & 0.40 & 0.51 & 0.41 \\
\hline
\end{tabular}

(b) Jezebel criticality to plutonium-239 nuclear data

\begin{tabular}{ccccc}
\hline & Reference & Eq. (13) & \multicolumn{2}{c}{ Eq. (14) } \\
& & $(I=400)$ & $(I=400)$ & $(I=1,600)$ \\
\hline$\sigma_{f}$ & 0.44 & 0.44 & 0.52 & 0.44 \\
$\sigma_{c}$ & 0.08 & 0.08 & 0.29 & 0.13 \\
$\bar{\nu}$ & 0.21 & 0.21 & 0.30 & 0.22 \\
$\sigma_{e}$ & 0.22 & 0.22 & 0.34 & 0.22 \\
$\sigma_{i e}$ & 0.29 & 0.28 & 0.33 & 0.30 \\
$\bar{\mu}$ & 0.16 & 0.16 & 0.24 & 0.16 \\
\hline
\end{tabular}

(c) Flattop-25 criticality to uranium-235 nuclear data

\begin{tabular}{ccccc}
\hline & Reference & $\begin{array}{c}\text { Eq. (13) } \\
(I=400)\end{array}$ & \multicolumn{2}{c}{ Eq. (14) } \\
\hline$\sigma_{f}$ & 0.29 & 0.28 & 0.54 & 0.34 \\
$\sigma_{c}$ & 0.19 & 0.17 & 0.43 & 0.23 \\
$\bar{\nu}$ & 0.24 & 0.24 & 0.37 & 0.28 \\
$\sigma_{e}$ & 0.11 & 0.11 & 0.34 & 0.23 \\
$\sigma_{i e}$ & 0.26 & 0.26 & 0.41 & 0.30 \\
$\bar{\mu}$ & 0.14 & 0.14 & 0.30 & 0.18 \\
\hline
\end{tabular}

(d) Flattop-25 criticality to uranium-238 nuclear data

\begin{tabular}{ccccc}
\hline & Reference & $\begin{array}{c}\text { Eq. (13) } \\
(I=400)\end{array}$ & \multicolumn{2}{c}{ Eq. (14) } \\
& & $(I=400)$ & $(I=1,600)$ \\
\hline$\sigma_{f}$ & 0.04 & 0.05 & 0.57 & 0.18 \\
$\sigma_{c}$ & 0.08 & 0.08 & 0.49 & 0.15 \\
$\bar{\nu}$ & 0.05 & 0.05 & 0.18 & 0.12 \\
$\sigma_{e}$ & 0.37 & 0.36 & 0.55 & 0.41 \\
$\sigma_{i e}$ & 0.46 & 0.44 & 0.44 & 0.46 \\
$\bar{\mu}$ & 0.31 & 0.30 & 0.29 & 0.33 \\
\hline
\end{tabular}




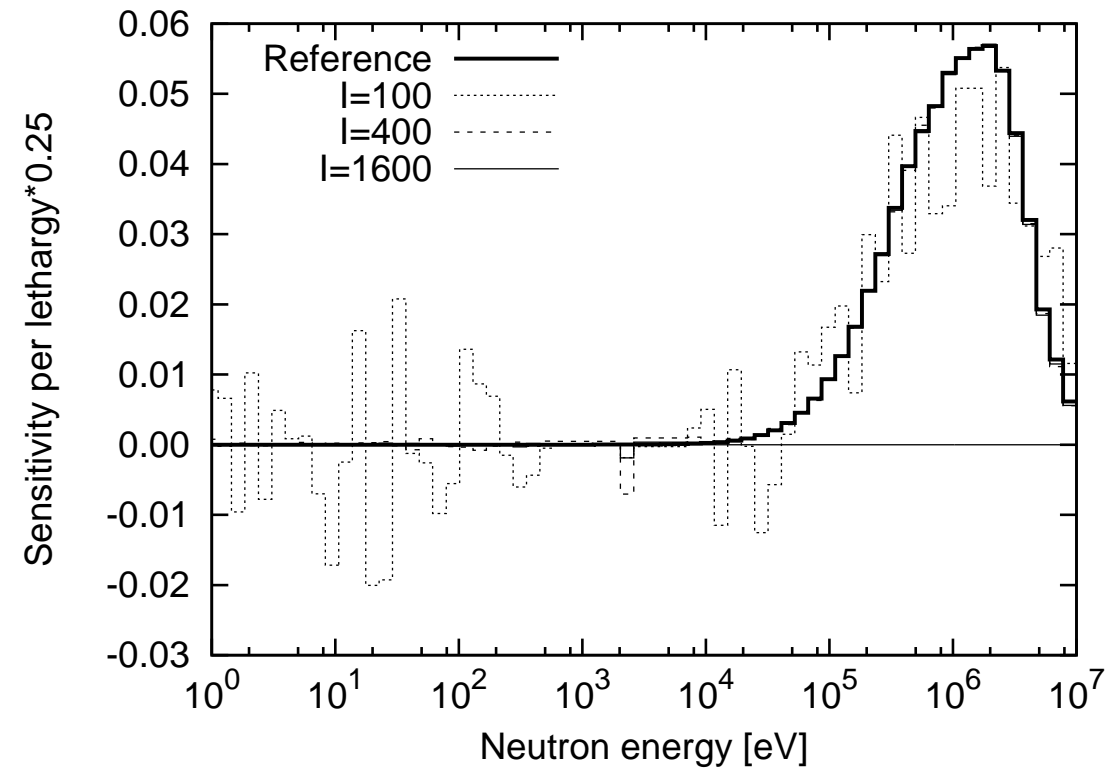

Figure 1: Sensitivities of Godiva criticality to uranium-235 fission cross section. Sample covariance matrix is used. The notation $I$ denotes the number of samples. 


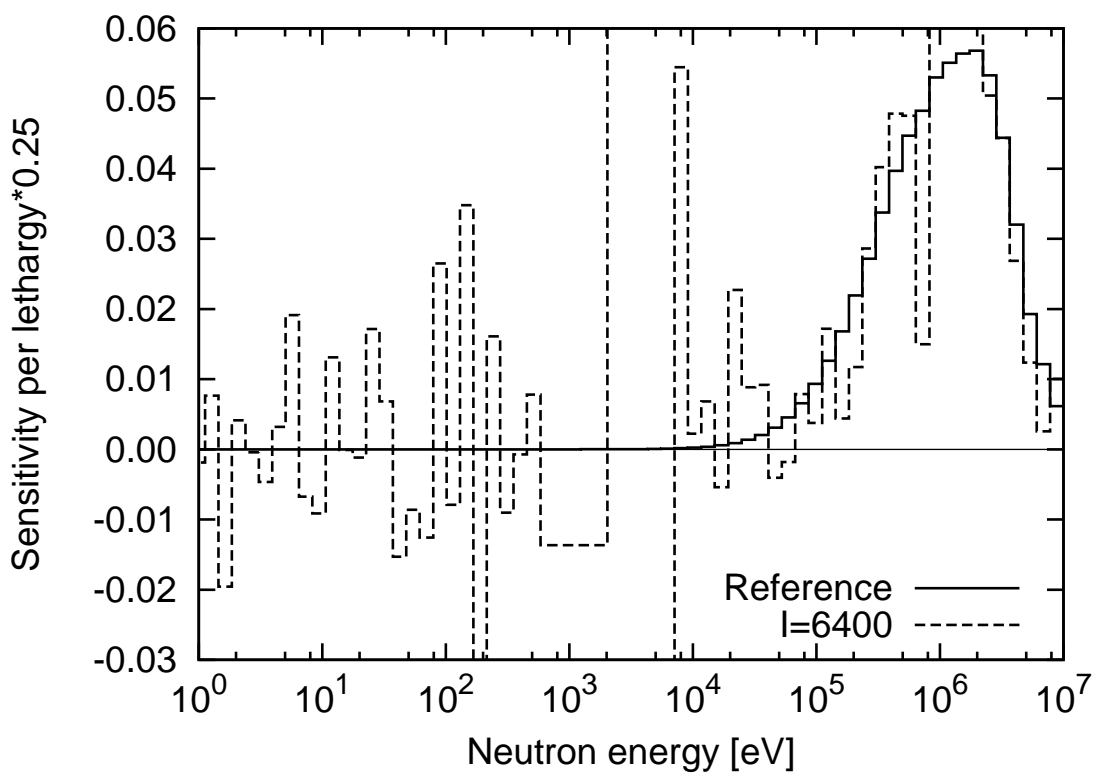

Figure 2: Sensitivities of Godiva criticality to uranium-235 fission cross section. True covariance matrix is used. 

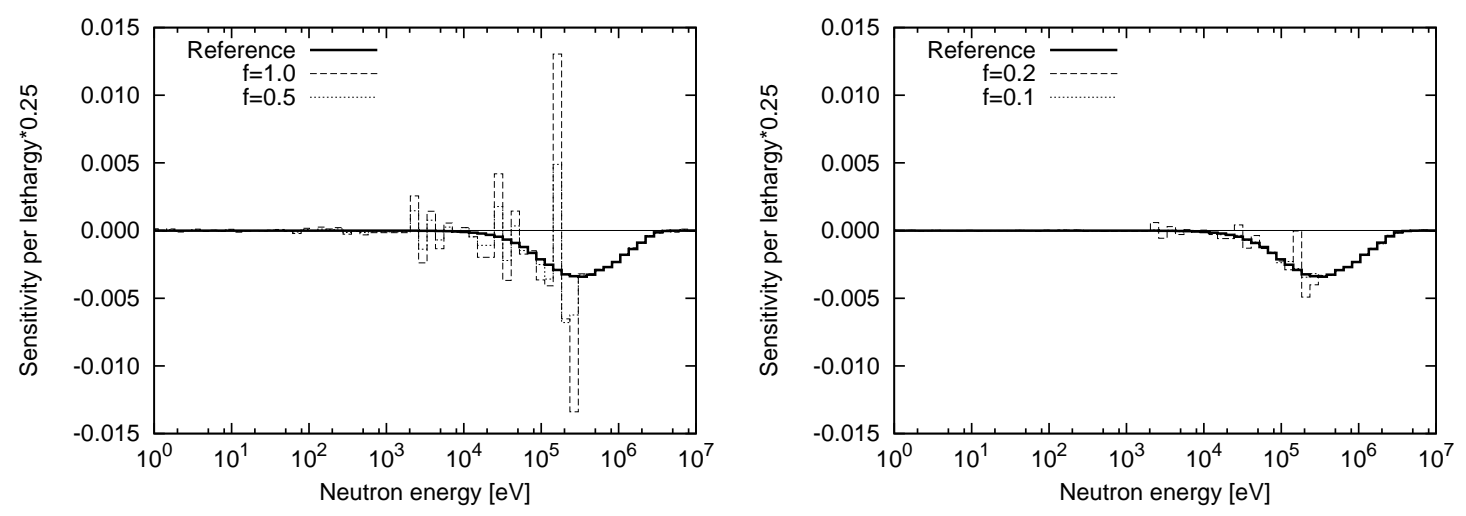

Figure 3: Sensitivities of Godiva criticality to uranium-235 capture cross section. All the elements of covariance matrices are multiplied by $f^{2}$. 


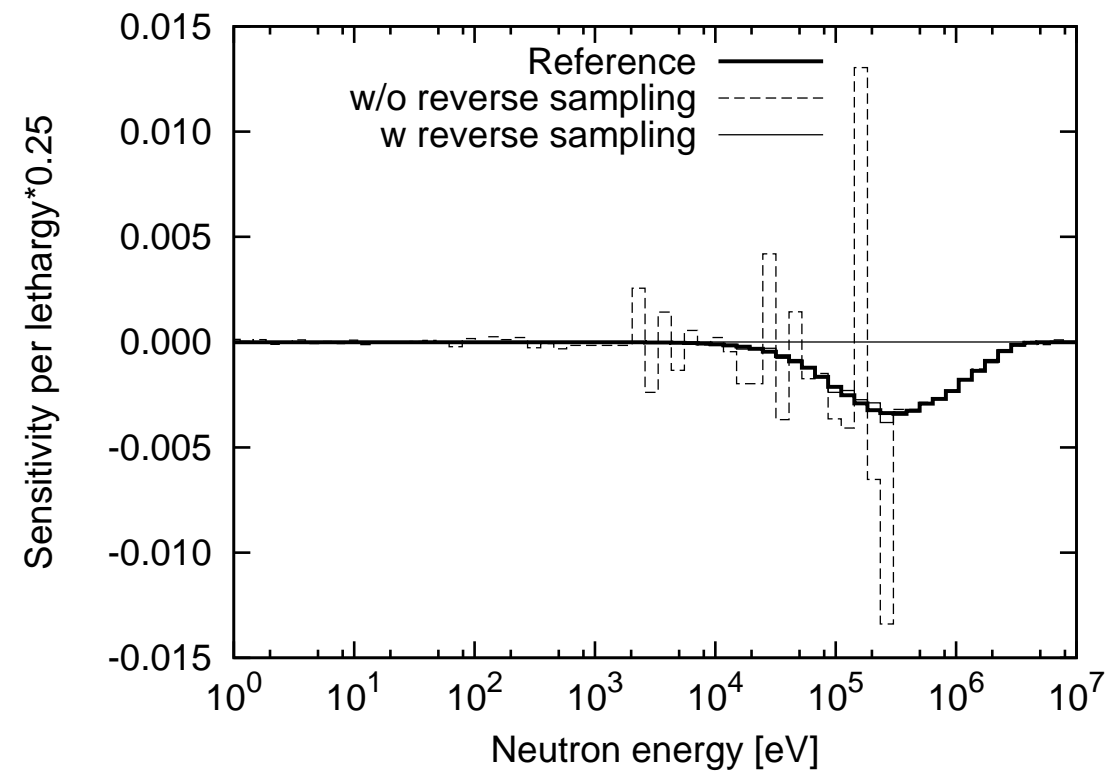

Figure 4: Sensitivities of Godiva criticality to uranium-235 capture cross section obtained with/without the reverse sampling method. 

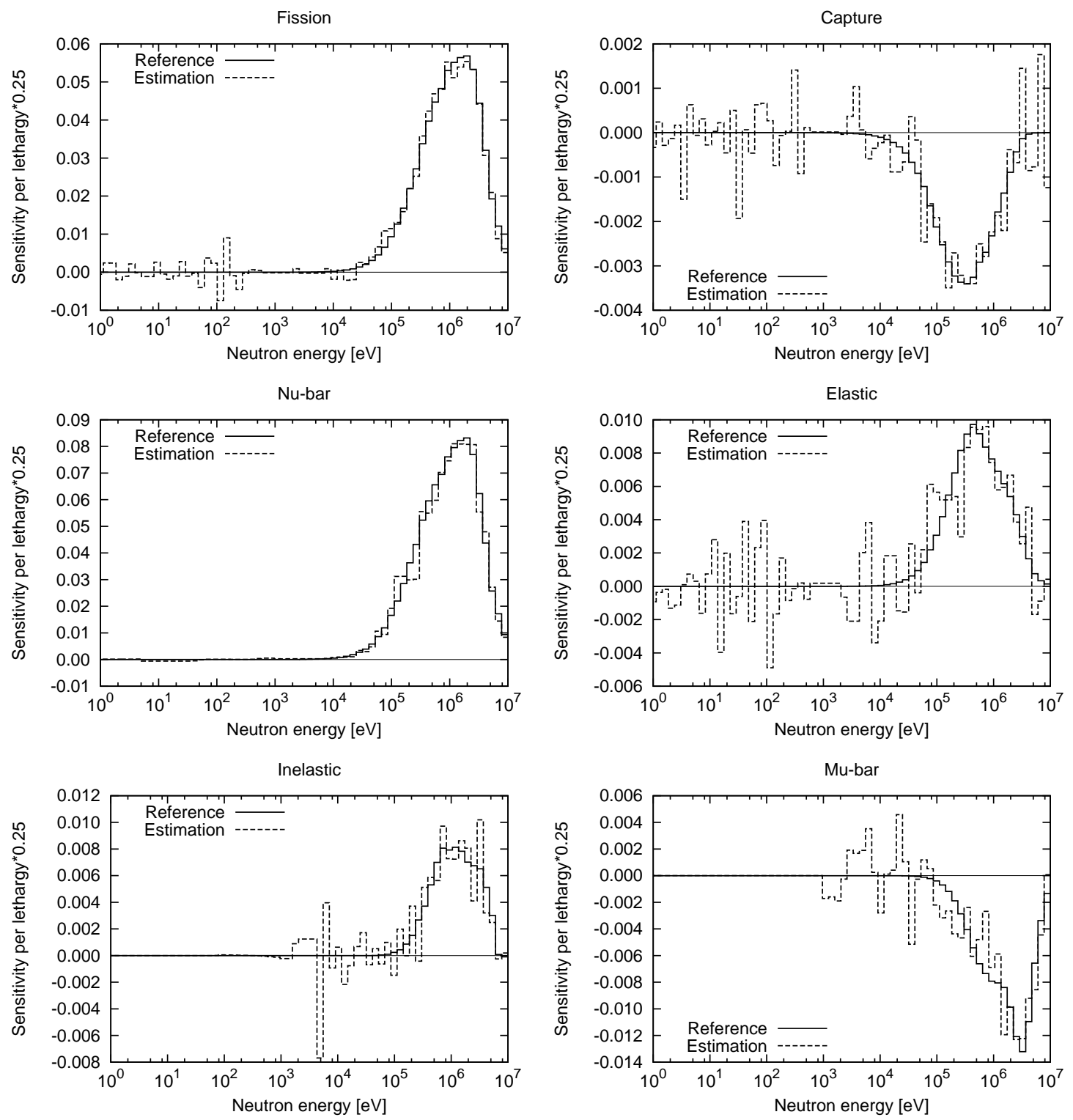

Figure 5: Sensitivity of Godiva criticality to uranium-235 nuclear data. 400 samples are used. 

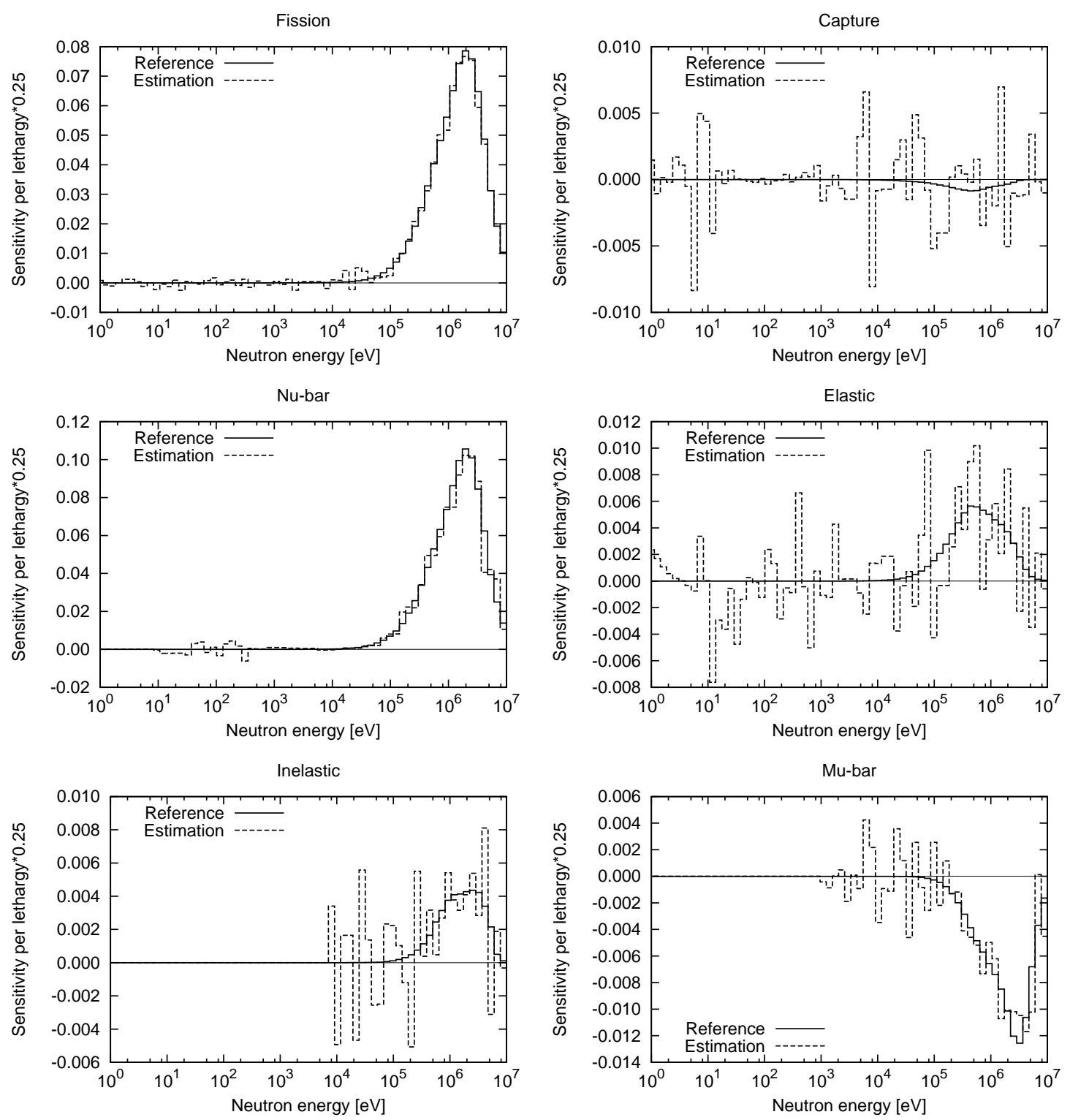

Figure 6: Sensitivity of Jezebel criticality to plutonium-239 nuclear data. 400 samples are used. 

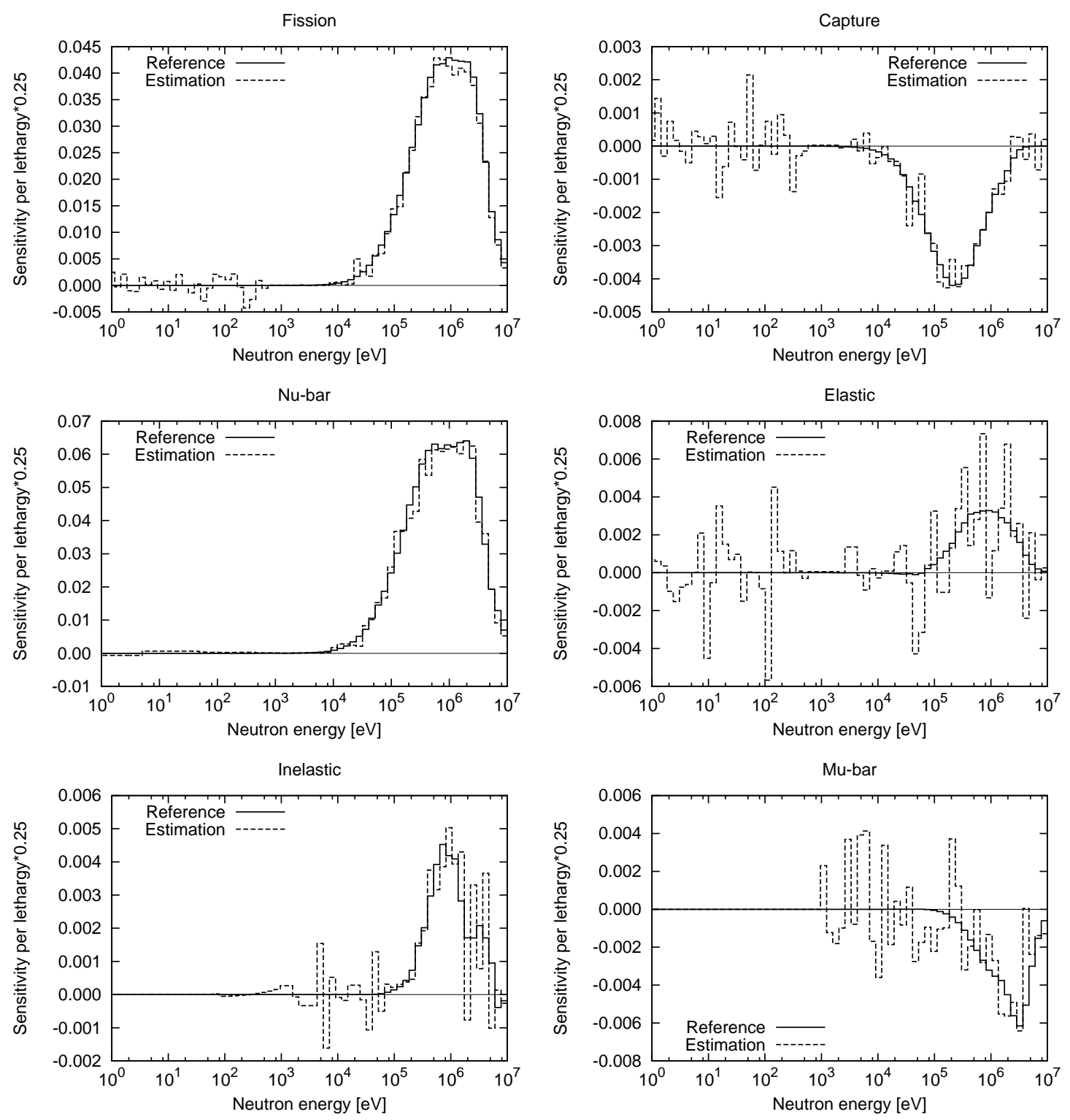

Figure 7: Sensitivity of Flattop-25 criticality to uranium-235 nuclear data. 800 samples are used. 
Fission

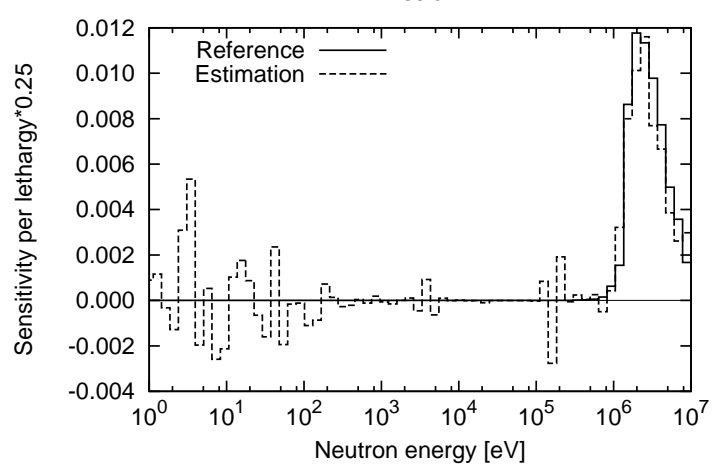

Nu-bar
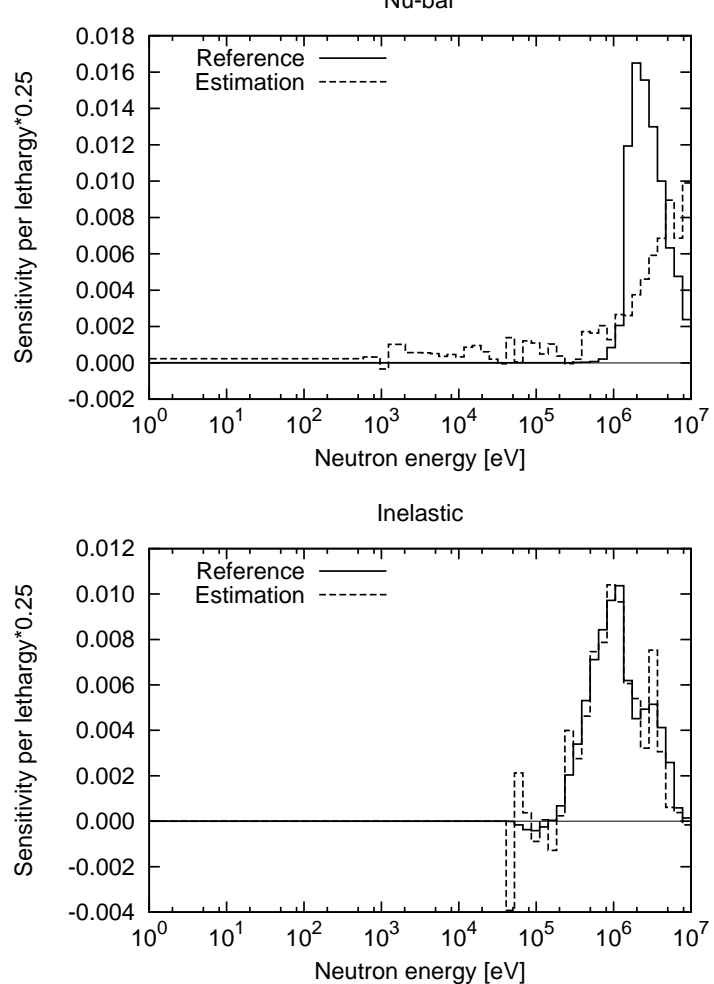

Capture

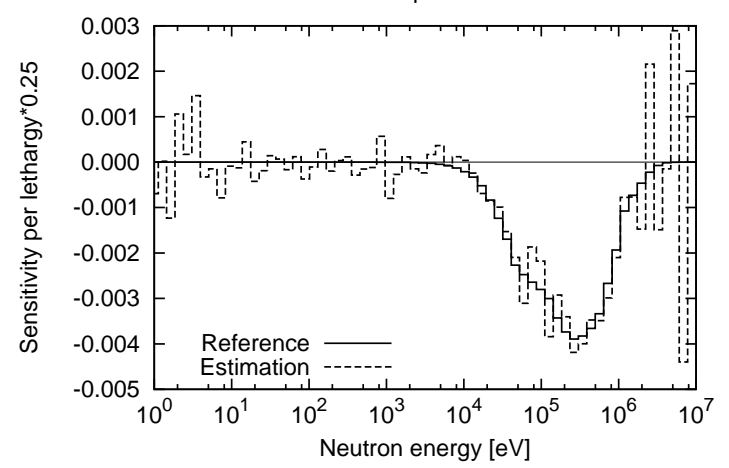

Elastic
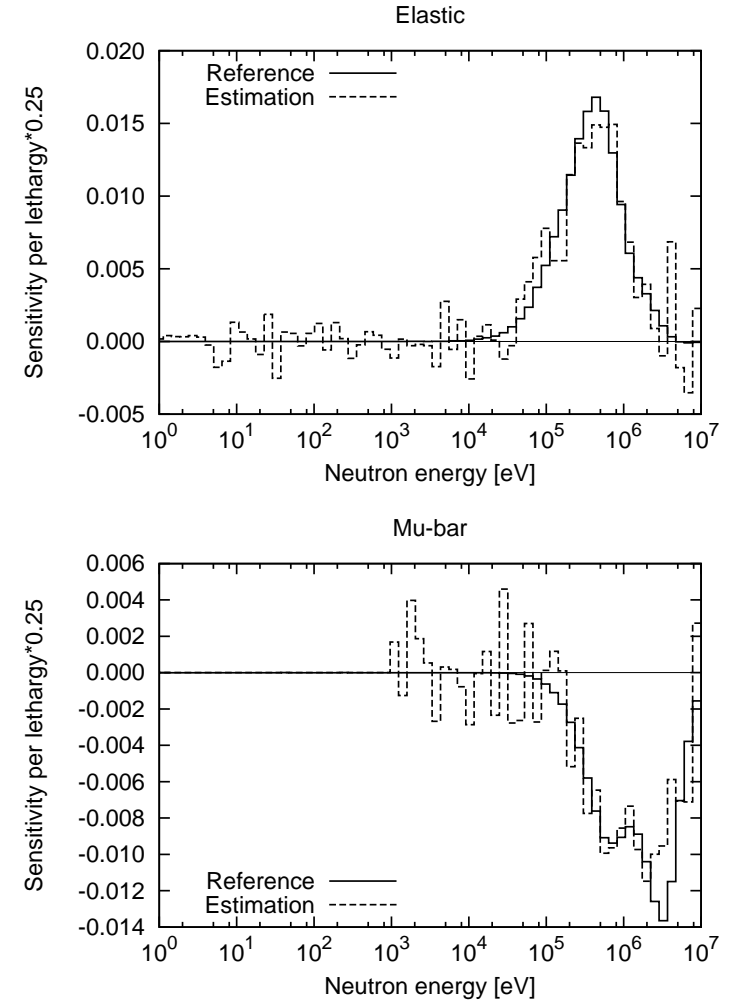

Figure 8: Sensitivity of Flattop-25 criticality to uranium-238 nuclear data. 800 samples are used. 


\section{List of Figure Captions}

Fig.1 Sensitivities of Godiva criticality to uranium-235 fission cross section. Sample covariance matrix is used. The notation $I$ denotes the number of samples.

Fig.2 Sensitivities of Godiva criticality to uranium-235 fission cross section. True covariance matrix is used.

Fig.3 Sensitivities of Godiva criticality to uranium-235 capture cross section. All the elements of covariance matrices are multiplied by $f^{2}$.

Fig.4 Sensitivities of Godiva criticality to uranium-235 capture cross section obtained with/without the reverse sampling method.

Fig.5 Sensitivity of Godiva criticality to uranium-235 nuclear data. 400 samples are used.

Fig.6 Sensitivity of Jezebel criticality to plutonium-239 nuclear data. 400 samples are used.

Fig.7 Sensitivity of Flattop-25 criticality to uranium-235 nuclear data. 800 samples are used.

Fig.8 Sensitivity of Flattop-25 criticality to uranium-238 nuclear data. 800 samples are used. 